\title{
PROTONS FROM THE ALPHA-PARTICLE BOMBARDMENT OF ${ }^{23} \mathrm{Na}$
}

\author{
by J. KUPERUS
}

Fysisch Laboratorium der Rijksuniversiteit te Utrecht, Nederland

\section{Synopsis}

Resonances in the yield of ground-state protons from alpha-particle bombardment of ${ }^{23} \mathrm{Na}$ were investigated in the energy range $E_{\alpha}=1.0-3.3 \mathrm{MeV}$. At least thirty-eight resonances were observed. Resonance energies and strengths are presented. At nine resonances angular distribution measurements lead to unique values of the spin of the resonance level. In two of these cases the parity of the resonance level was also determined. In two other cases the angular distribution left two possibilities for the resonance spin. Angular distributions at ten other resonances could not be treated with a single level analysis.

1. Introduction. The investigation of resonances in alpha-proton reactions in the $Z=10-20$ region was started with a study of the ${ }^{31} \mathrm{P}(\alpha, p)^{34} \mathrm{~S}$ reaction, reported in a previous paper ${ }^{1}$ ).

In the present experiment ground-state protons of the ${ }^{23} \mathrm{Na}(\alpha, p)^{26} \mathrm{Mg}$ reaction were observed. In this reaction the ground state of the target nucleus has $J^{\pi}=3 / 2^{+}$, permitting orbital momentum mixing in the formation channel, which makes the analysis of the measurements less simple than in the reaction mentioned above, where the target nucleus has $J^{\pi}=1 / 2^{+}$, excluding orbital momentum mixing. Moreover the higher density and larger width of the resonance levels may give rise to interference, distorting single level angular distributions.

In the region up to $E_{\alpha}=3.3 \mathrm{MeV}$ thirty-eight resonances were observed, of which twenty-one were sufficiently resolved to make an angular distribution measurement meaningful. In eleven cases no interference effects were observed. At the other ten resonances the angular distribution was certainly influenced by contributions from other levels.

The experimental equipment is briefly described in section 2 . The method of analysis and the results are presented in sections 3 and 4, respectively.

2. Experimental. Alpha particles accelerated with the Utrecht $3 \mathrm{MV}$ Van de Graaff generator bombarded targets of $\mathrm{NaCl}$, evaporated on a solid copper backing. Target thicknesses varying from $80 \mathrm{up}$ to $1000 \mu \mathrm{g} / \mathrm{cm}^{2}$ were used during the experiment. 
The target was surrounded by six silicon surface-barrier counters at laboratory angles of $172,150,135,120,87$ and 40 degrees with respect to the beam. Angular distribution measurements were performed with the five detectors in backward directions, the angle between target and beam being $45^{\circ}$. For the investigation of the forward-backward symmetry, the target was given an angle of $20^{\circ}$ with respect to the beam. The yield of the $40^{\circ}$ counter was then compared with the result from the other five detectors.

A more extensive description of the experimental set-up has been given in earlier papers $\left.{ }^{1}{ }^{2}\right)$.

3. Analysis. Yields. The thick target yield of a resonance is proportional to the resonance strength $(2 J+1) \Gamma_{p} \Gamma_{\alpha} / \Gamma$, which easily leads to lower limits for the particle widths $\Gamma_{\alpha}, \Gamma_{p}$ and for the dimensionless reduced alpha-particle width $\theta_{\alpha}^{2}$ of the resonance level $\left.{ }^{1}\right)^{2}$ ). The Coulomb barrier penetration being less probable for the incoming alpha particles than for the outgoing protons, the lower limit for the proton width is of less interest.

In energy regions, having a resonance density too high to permit a thick target measurement, the strengths were determined by a comparison of the area under a thin target resonance curve with the area under a resonance curve, taken with the same thin target at a resonance of which the yield was known from a thick target measurement.

Angular distributions. The ground state of the target nucleus has $J=3 / 2$, which is equal to the channel spin of the incoming channel. This allows orbital momentum mixing for the incoming alpha particles. The proton orbital momentum is uniqucly determined by the spin and parity of the resonance level. The angular distribution at an isolated resonance with a certain spin and parity is thus determined by only one parameter, the orbital momentum amplitude mixing ratio $\varepsilon$ in the alpha-particle channel.

The theoretical angular distributions $W\left(J^{\pi}, l_{\alpha}, l_{\alpha}^{\prime}, \theta\right)$ for alpha-particle angular momenta up to $l_{\alpha}=5$ are presented in table $\mathrm{I}$. The expected angular distribution at a certain isolated resonance, having spin and parity $J^{\pi}$, which can be formed by alpha-particle orbital momenta $l_{\alpha}$ and $l_{\alpha}^{\prime}$ is then given by

$$
W(\theta)=\frac{W\left(J^{\pi}, l_{\alpha}, l_{\alpha}, \theta\right)+\varepsilon^{2} W\left(J^{\pi}, l_{\alpha}^{\prime}, l_{\alpha}^{\prime}, \theta\right)+2 \varepsilon \cos \varphi_{l_{\alpha} l_{\alpha}} W\left(J^{\pi}, l_{\alpha}, l_{\alpha}^{\prime}, \theta\right)}{\left(1+\varepsilon^{2}\right)}
$$

In this formula $\varepsilon$ is the amplitude ratio and $\varphi_{l_{\alpha} l_{\alpha^{\prime}}}$ the Coulomb phase difference of the two possible orbital momenta.

After background subtraction, the observed angular distribution was converted to the center-of-mass system. These data were then fitted to the possible one-parameter range of curves for each value of $J^{\pi}$, determining the 
value of $\varepsilon$ with minimum $\chi^{2}$. Values of $J^{\pi}$, having a $\chi^{2}$ above the $0.1 \%$ probability limit for all values of $\varepsilon$ were supposed to be excluded. In this way, the possible values of $J^{\pi}$ and the corresponding values of $\varepsilon$, as well as the Legendre polynomial coefficients of the angular distribution, could be determined for isolated resonances. The errors in the $\varepsilon$ values and in the Legendre coefficients were also calculated, using the statistical error in the observed number of counts, increased with an error of $1.5 \%$ in the detector solid angle. These calculations were carried out with the Utrecht University ZEBRA and X1 computers, using the program mentioned in ref. 2.

TABLE I

\begin{tabular}{|c|c|c|c|c|c|c|c|c|c|}
\hline \multicolumn{10}{|c|}{$\begin{array}{c}\text { Theoretical }{ }^{23} \mathrm{Na}(\alpha, p)^{26} \mathrm{Mg} \text { angular distribution Legendre polynomial coefficients for different } \\
\text { values of the alpha-particle and proton orbital momenta, } l_{\alpha} \text { and } l_{p} \text {, respectively, and for } \\
\text { different resonance spins and parities, } J^{\pi}\end{array}$} \\
\hline$l_{\alpha}$ & $l_{\alpha}^{\prime}$ & $J^{\pi}$ & $l_{p}$ & $A_{0}$ & $A_{2}$ & $A_{4}$ & $A_{6}$ & $A_{8}$ & $A_{10}$ \\
\hline 1 & 1 & $1 / 2^{-}$ & 1 & 1 & & & & & \\
\hline 2 & 2 & $1 / 2^{+}$ & 0 & 1 & & & & & \\
\hline 1 & 1 & $3 / 2-$ & 1 & 1 & -0.800 & & & & \\
\hline 3 & 3 & & & 1 & +0.800 & & & & \\
\hline 1 & 3 & & & 0 & -0.600 & & & & \\
\hline 0 & 0 & $3 / 2+$ & 2 & 1 & & & & & \\
\hline 2 & 2 & & & 1 & 0.000 & & & & \\
\hline 0 & 2 & & & 0 & -1.000 & & & & \\
\hline 1 & 1 & $5 / 2^{-}$ & 3 & 1 & +0.800 & & & & \\
\hline 3 & 3 & & & 1 & +0.629 & -0.429 & & & \\
\hline 1 & 3 & & & 0 & -0.420 & -1.050 & & & \\
\hline 2 & 2 & $5 / 2^{+}$ & 2 & 1 & +0.408 & -0.980 & & & \\
\hline 4 & 4 & & & 1 & +1.020 & +0.551 & & & \\
\hline 2 & 4 & & & 0 & -0.300 & -0.750 & & & \\
\hline 3 & 3 & $7 / 2-$ & 3 & 1 & +0.794 & -0.117 & -1.010 & & \\
\hline 5 & 5 & & & 1 & +1.111 & +0.818 & +0.404 & & \\
\hline 3 & 5 & & & 0 & -0.177 & -0.523 & -0.791 & & \\
\hline 2 & 2 & $7 / 2^{+}$ & 4 & 1 & +1.020 & +0.551 & & & \\
\hline 4 & 4 & & & 1 & +0.884 & +0.150 & -0.606 & & \\
\hline 2 & 4 & & & 0 & -0.228 & -0.672 & -1.016 & & \\
\hline 3 & 3 & $9 / 2^{-}$ & 5 & 1 & +1.111 & +0.818 & +0.404 & & \\
\hline 5 & 5 & & & 1 & +1.010 & +0.504 & -0.162 & -0.685 & \\
\hline 3 & 5 & & & 0 & -0.143 & -0.445 & -0.800 & -0.969 & \\
\hline 4 & 4 & $9 / 2^{+}$ & 4 & 1 & +0.964 & +0.360 & -0.419 & -0.997 & \\
\hline 5 & 5 & $11 / 2-$ & 5 & 1 & +1.054 & +0.633 & +0.033 & -0.597 & -0.970 \\
\hline 4 & 4 & $11 / 2^{+}$ & 6 & 1 & +1.157 & +0.961 & +0.661 & +0.312 & \\
\hline 5 & 5 & $13 / 2^{-}$ & 7 & 1 & +1.183 & +1.046 & +0.823 & +0.545 & +0.249 \\
\hline
\end{tabular}

This analysis neglects contributions in the angular distributions caused by interference of resonance levels, which would introduce additional, strongly energy dependent terms. When a resonance is not sufficiently isolated, the angular distribution at a certain energy will be dependent on the distances and the widths of all contributing levels, as well as on the individual orbital momentum mixing ratios. Interfering resonances with 
opposite parity will introduce a deviation from the $\theta=90^{\circ}$ symmetry. No attempts were made to interpret angular distributions at resonances, showing such interference effects.

4. Results A yield curve of the ground-state protons from the ${ }^{23} \mathrm{Na}(\alpha, p)^{26} \mathrm{Mg}$ reaction for the $E_{\alpha}=1.8-3.3 \mathrm{MeV}$ region, showing at least thirty-eight resonances, is presented in fig. 1 . Some more unresolved resonance structure

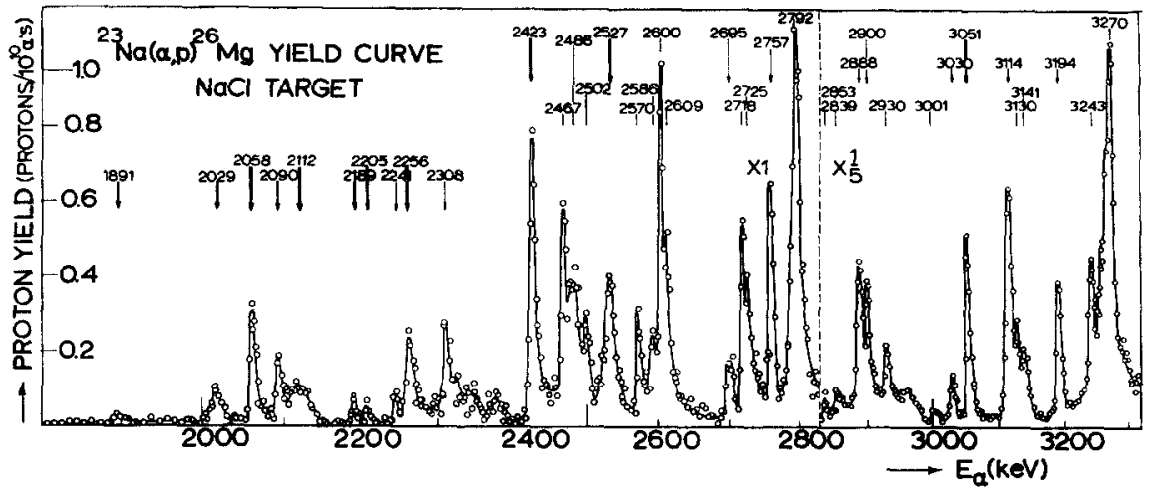

Fig. 1. Yield curve for the ${ }^{23} \mathrm{Na}(\alpha, p)^{20} \mathrm{Mg}$ reaction. The sum of the number of counts, observed at laboratory angles of $87,120,135,150$, and 172 degrees is plotted. The angular distribution at resonances, indicated with an arrow, was investigated. Resonances with a thick arrow could be treated as isolated levels.

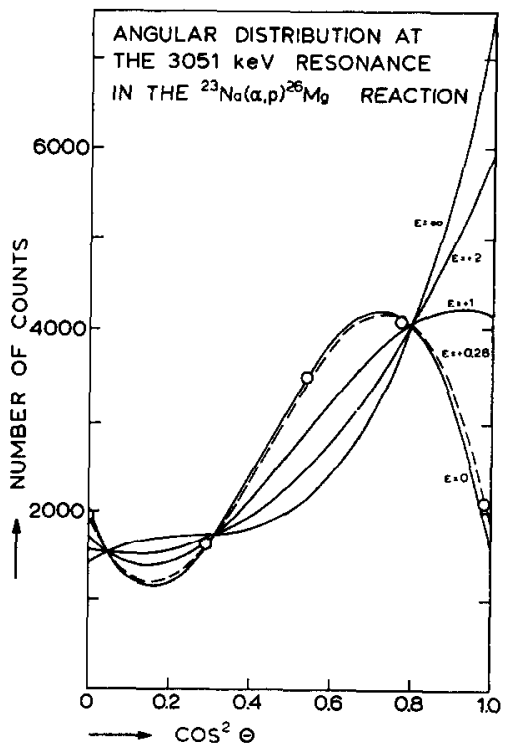

Fig. 2. Angular distribution observed at the $3051 \mathrm{keV}$ resonance in the ${ }^{23} \mathrm{Na}(\alpha, p)^{26} \mathrm{Mg}$ reaction. The solid curves represent the theoretical possibilities for a $7 / 2-$ resonance at this energy for some positive values of the amplitude mixing ratio $\varepsilon$. The dotted curve shows the best fit $(\varepsilon=0.28$ or $\varepsilon=-0.14)$. 
in this curve was too weak for further investigation. No resonances were observed in the $E_{\alpha}=1.0-1.8 \mathrm{MeV}$ region. Wigner limit considerations exclude the existence of observable resonances at still lower energies. Protons to the first excited state of ${ }^{26} \mathrm{Mg}$, also observed as a peak in the energy spectrum, were not investigated.

Angular distribution measurements were made at twenty-one resonances, indicated with an arrow in fig. 1. The appearance of angular distribution energy dependence and/or deviation from forward-backward symmetry was investigated at those resonances, having an angular distribution, satisfying a single level analysis, but which did not appear to be isolated in the resonance curve. In this way it was shown that a single level analysis was not meaningful at ten resonances, whereas the other eleven resonances, indicated with a thick arrow in fig. 1, could be treated as isolated levels.

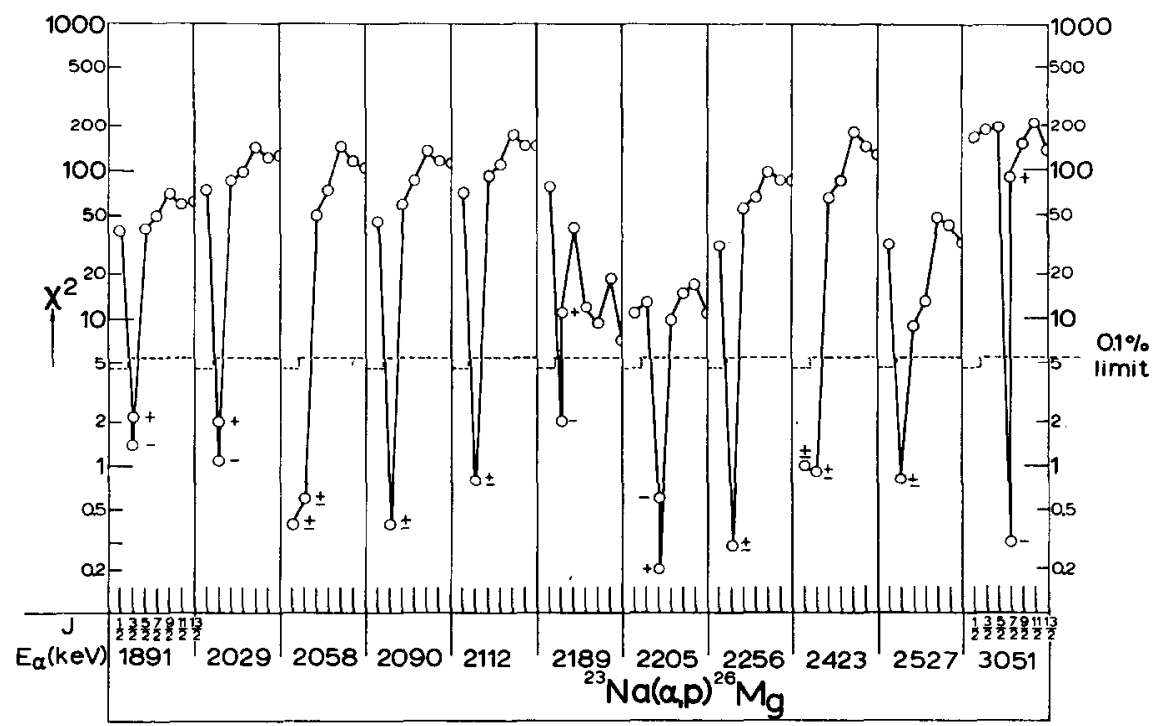

Fig. 3. Angular distribution $\chi^{2}$ values for different $J^{\pi}$ values of the resonance level at eleven isolated resonances. When for a certain spin the minimum value of $\chi^{2}$ exceeds the $0.1 \%$ probability limit for both parities, only the lowest of the two values is plotted.

As an example of the analysis at an isolated resonance, fig. 2 shows the angular distribution observed at the $3051 \mathrm{keV}$ resonance, together with the range of curves, which is theoretically possible at this energy for a $7 / 2-$ resonance. The curves are shown for some positive values of the amplitude mixing ratio $\varepsilon$ for $f$ and $h$ alpha particles. The same range of curves holds for negative values of $\varepsilon$. The very large $A_{6}$ term in the angular distribution is an unique feature of this resonance.

The values of $\chi^{2}$ as calculated from the experimental distributions for 


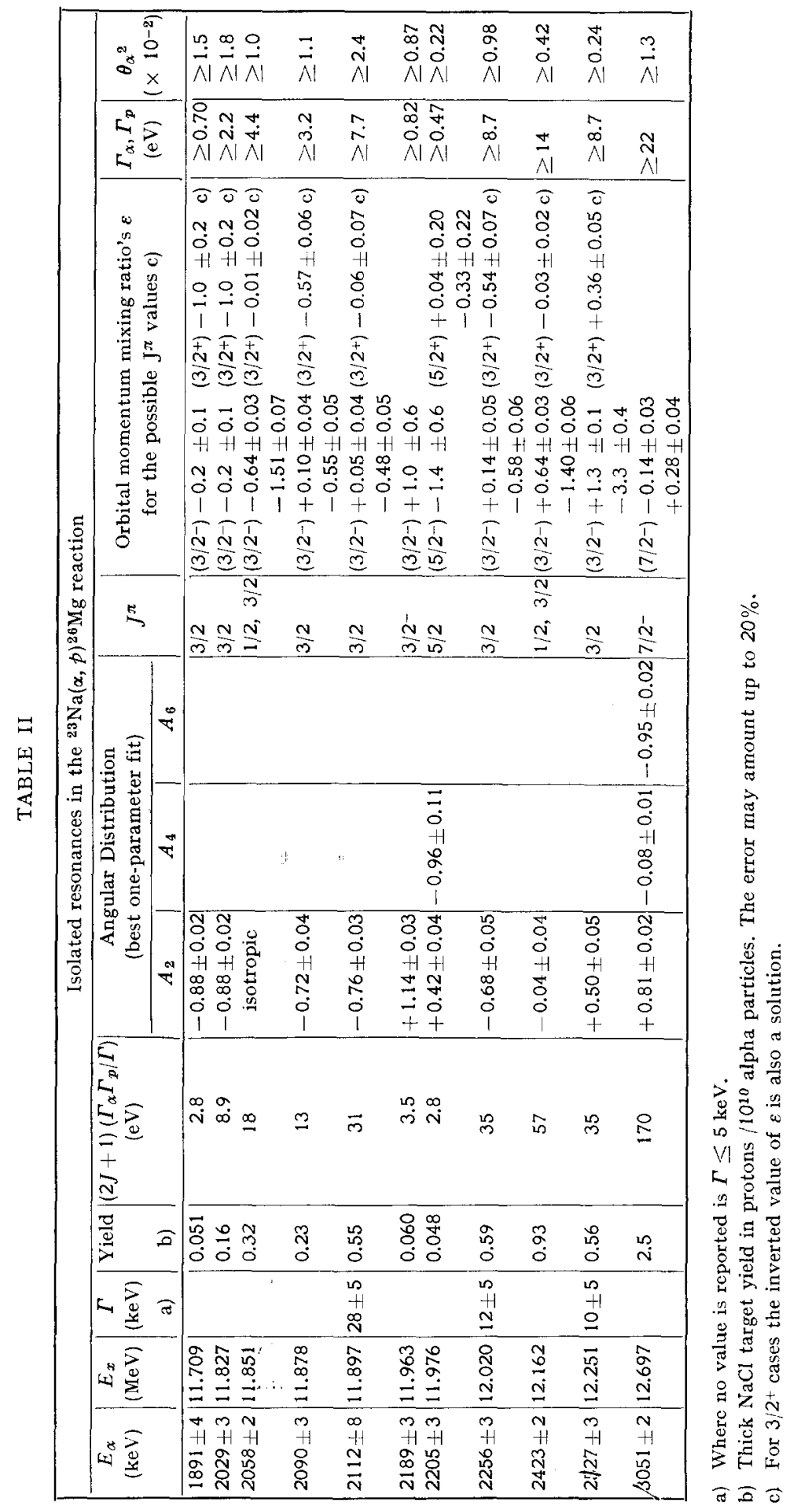


assumed spin values $1 / 2$ through $13 / 2$ at the eleven isolated resonances are plotted in fig. 3. When for a certain spin the minimum value of $\chi^{2}$ exceeds the $0.1 \%$ limit for both parities only the lowest of the two values is plotted. Wigner limit reasons exclude the assignment of spin values higher than $13 / 2$ at any of these resonances.

TABLE III

\begin{tabular}{|c|c|c|c|c|c|c|c|}
\hline \multicolumn{8}{|c|}{$\begin{array}{c}\text { Resonances, investigated in the }{ }^{23} \mathrm{Na}(\alpha, p)^{26} \mathrm{Mg} \text { reaction, } \\
\text { which can not be treated as isolated levels }\end{array}$} \\
\hline \multirow{2}{*}{$\begin{array}{c}E_{\alpha} \\
(\mathrm{keV})\end{array}$} & \multirow{2}{*}{$\begin{array}{c}E_{x} \\
(\mathrm{MeV})\end{array}$} & \multirow{2}{*}{$\begin{array}{c}\Gamma \\
(\mathrm{keV}) \\
\text { a) }\end{array}$} & \multirow{2}{*}{$\begin{array}{l}\text { Yield } \\
\text { b) }\end{array}$} & \multirow{2}{*}{$\begin{array}{c}2 J+1)\left(\Gamma_{\alpha} \Gamma_{p} / \Gamma\right. \\
(\mathrm{eV})\end{array}$} & \multicolumn{3}{|c|}{$\begin{array}{l}\text { Main even terms in the } \\
\text { angular distribution }\end{array}$} \\
\hline & & & & & $A_{2}$ & $A_{4}$ & $A_{6}$ \\
\hline $2241 \pm 3$ & 12.007 & & 0.10 & 5.9 & -0.3 & -0.4 & \\
\hline $2308 \pm 3$ & 12.064 & & 0.23 & 14 & +1.1 & -0.6 & -0.7 \\
\hline $2695 \pm 3 c, d)$ & 12.394 & $9 \pm 5$ & 0.31 & 20 & -0.5 & & \\
\hline $2757 \pm 3$ & 12.447 & & 0.73 & 47 & +0.2 & -0.9 & +0.2 \\
\hline $2792 \pm 5 \mathrm{c})$ & 12.476 & $13 \pm 5$ & 2.8 & 180 & -0.7 & +0.4 & \\
\hline $2888 \pm 5 \mathrm{cl}$ & 12.558 & $7 \pm 4$ & 4.2 & 280 & -0.5 & & \\
\hline $2900 \pm 3 \mathrm{c})$ & 12.568 & & 1.0 & 70 & +0.3 & & \\
\hline $3030 \pm 3$ & 12.679 & & 0.55 & 37 & +0.9 & -0.8 & -0.3 \\
\hline $3114 \pm 3 c)$ & 12.751 & $11 \pm 5$ & 6.4 & 440 & +0.1 & +0.1 & \\
\hline $3194 \pm 3 c, d)$ & 12.819 & & 2.7 & 190 & -0.4 & & \\
\hline
\end{tabular}

a) Where no value is reported, $\Gamma \leq 5 \mathrm{keV}$.

b) Thick target yield in protons $/ 10^{10}$ alpha particles.

The error may amount up to $20 \%$.

c) The angular distribution shows energy dependence.

d) The angular distribution shows deviation from forward-backward symmetry.

TARLE IV

\begin{tabular}{|l|c|c|c|}
\hline \multicolumn{3}{|c|}{ Other resonances in the ${ }^{23} \mathrm{Na}(\alpha, p)^{26} \mathrm{Mg}$ reaction } \\
\hline $\begin{array}{c}E_{\alpha} \\
(\mathrm{keV})\end{array}$ & $\begin{array}{c}E_{x} \\
(\mathrm{MeV})\end{array}$ & $\begin{array}{c}\text { Yield } \\
\text { a) }\end{array}$ & $\begin{array}{c}(2 J+1) \Gamma_{\alpha} \Gamma_{p} / \Gamma \\
(\mathrm{eV})\end{array}$ \\
\hline $2467 \pm 3$ & 12.200 & 0.41 & 25 \\
$2486 \pm 5 \mathrm{~b})$ & 12.216 & 0.49 & 30 \\
$2502 \pm 5$ & 12.229 & 0.20 & 12 \\
$2570 \pm 3$ & 12.287 & 0.18 & 11 \\
$2586 \pm 3$ & 12.301 & 0.17 & 10 \\
$2600 \pm 3$ & 12.313 & 1.35 & 84 \\
$2609 \pm 5$ & 12.321 & 0.33 & 21 \\
$2718 \pm 5$ & 12.413 & 0.82 & 53 \\
$2752 \pm 5$ & 12.442 & 0.30 & 19 \\
$2839 \pm 3$ & 12.517 & 0.10 & 6.3 \\
$2853 \pm 5$ & 12.528 & 0.65 & 43 \\
$2930 \pm 5$ & 12.594 & 0.94 & 63 \\
$3001 \pm 5 \mathrm{~b})$ & 12.655 & 0.13 & 8.8 \\
$3130 \pm 5$ & 12.764 & 0.67 & 46 \\
$3141 \pm 5$ & 12.777 & 0.50 & 34 \\
$3243 \pm 5$ & 12.861 & 2.9 & 200 \\
$3270 \pm 8$ b) & 12.884 & 14 & 970 \\
\hline
\end{tabular}

a) Thick target yield in protons/1010 alpha particles, not corrected for angular distribution effects. The error may amount up to $20 \%$.

b) Probably broad and/or double. 
The results obtained at these resonances are given in table II. It presents the resonance energy, the excitation energy in $\left.{ }^{27} \mathrm{Al}\left(E_{b}=10.098 \mathrm{MeV}{ }^{3}\right)\right)$, the width, the thick target yield, the resonance strength, the angular distribution coefficients giving the best fit for any spin and parity of the resonance level, the possibilities for spin and parity of the resonance level and for the orbital momentum mixing ratio. The last columns show lower limits for particle widths and reduced alpha particle widths.

The other ten resonances investigated did all show indications that a single level analysis, was not allowed. Table III gives resonance energies, excitation energies, widths, yields, and resonance strengths as well as the main even terms in the angular distribution.

Energies, excitation energies, and yields for seventeen other resonances, insufficiently resolved for an angular distribution measurement, are given in table IV. The yields for these resonances as determined with the five counters in the backward directions are not corrected for angular distribution effects.

Acknowledgement. This investigation was partly supported by the joint research program of the "Stichting voor Fundamenteel Onderzoek der Materie" and the "Nederlandse Organisatie voor Zuiver Wetenschappelijk Onderzoek".

Considerable assistence in taking the data given by W. Hogervorst is gratefully acknowledged. The author is much indebted to Prof. P. M. Endt and Dr. C. van der Leun for their help and their suggestions in the preparation of this article.

Received 12-6-64

\section{REFERENCES}

1) Kuperus, J., Physica 30 (1964) 899.

2) Kuperus, J., Glaudemans, P. W. M. and Endt, P. M., Physica 29 (1963) 1281.

3) Endt, P. M. and Van der Leun, C., Nucl. Phys. 3n (1962) 1. 